\section{The Farnsworth-Munsell 100-hue test: A BASIC program for scoring and analysis}

\author{
STANLEY COREN \\ University of British Columbia \\ Vancouver, British Columbia, Canada
}

The Farnsworth-Munsell 100-hue test (Farnsworth, 1943,1957 ) is one of the most popular tests for color discrimination. It also has been extensively used to determine the nature of color-discrimination losses by examining the pattern of color confusions (see Pokorny et al., 1981). The total error score is highly correlated with the matching range on the anomaloscope and with the wavelength discrimination function (a measure of the wavelength difference needed for an observer to detect a color difference), as shown by Lakowski (1971).

The test itself consists of 85 movable color samples arranged in four boxes of 21 or 22 colors each. These color samples represent perceptually equal steps of hue and form a natural hue circle. Two "pilot colors" are fixed at either end of each box, and the subject's task is to order the samples so that they form a perceptually continuous series of colors, gradually changing from the first pilot color to the second.

The scoring is mathematically simple; however, especially when there are many errors, scoring often is quite tedious and subject to human error. Scores are based upon the misplacement of color samples in the series. Error scores are calculated according to the distance between any pair of sample placements. This yields separate scores for each box and a total score. Determining the nature of any implied color deficit, however, requires the scorer to make several more passes, and usually requires plotting the error pattern.

Subsidiary analyses can provide information on the nature of any discrimination errors. Because there is a range of color samples which fall on the main confusion lines for each of the known and theoretical color deficiencies (see Lakowski, 1969), deficits may be classified by comparing the errors in each of these ranges. Furthermore, if the full error pattern is considered, an axis of confusion may be obtained. If the test is conducted using a CIE Source $\mathrm{C}$, the dominant wavelengths defining the confusion axis may also be found. These analyses are timeconsuming and, due to the many small calculations required, often are fraught with opportunities for error, especially when performed by minimally trained examiners.

\footnotetext{
This research was supported in part by a grant from the Natural Science and Engineering Research Council of Canada. I would like to thank Romuald Lakowski for his assistance. The author's mailing address is: Department of Psychology, University of British Columbia, Vancouver, British Columbia, Canada V6T IW5.
}

To simplify scoring of the Farnsworth-Munsell test and to provide some of the useful higher level analyses available, a BASIC language microcomputer program was created.

Requirements. The program is written in Applesoft BASIC. Although it was developed on a 48K Apple II+, it runs on all other members of the Apple family of microcomputers (IIe and IIc). Conversion to other dialects of BASIC should be quite simple, usually requiring only changes in the screen-clearing commands. A printer is desirable, since hard copy can then be obtained (options for subject identification on the output are provided), and also a histogram, providing a visual representation of the error pattern, will be automatically given when printed output is requested. When printed output is called for, the program assumes that the printer is in Slot 1. However, for most purposes, the screen output is quite adequate.

Inputs. The program is written in a user-friendly format. Complete instructions may be called up by the operator at the beginning of each scoring run. Successive screens prompt the user, indicating the inputs required or permitted. Most of the obvious erroneous inputs are screened out.

The user only needs to enter the runs of errors. Correct placements do not have to be scored, and correct boxes may be skipped. The input is organized around the usual score sheet used with the test, and for a fairly complex test result (yielding an error score of over 250), input times average between a minute and a minute and a half.

Outputs. The statistics include the scores for the individual boxes and the total score. In addition, separate scores are provided for the samples along the confusion lines for protanopes, deuteranopes, tritanopes, and teteranopes. Both totals and mean scores are provided for these ranges.

The program also computes the axes of confusion. These are given in terms of the sample numbers and also in terms of dominant wavelengths, which are valid if a nominal CIE Source $C$ illuminant was used in testing. The axes computations are only meaningful if there are sizable errors; hence, a threshold of four errors per sample in the confusion ranges has been set. If desired, the user may modify the program to alter this preset value.

The final data provided is a listing of the computed error scores for each color sample, so that further analyses or replotting may be performed. When printed output is requested, a histogram, providing the distribution of error scores by sample, is also given.

Availability. A program listing (rather fully annotated) is available without charge from the author. Copies of the program on diskette (DOS 3.3) may be obtained by sending a blank diskette plus $\$ 2$ to cover the cost of postage and handling. 


\section{REFERENCES}

FARNSWORTH, D. (1943). The Famsworth-Munsell 100-hue and dichotomous tests for color vision. Journal of the Optical Society of America, 33, 568-578.

FARNSWORTH, D. (1957). The Farnsworth-Munsell 100-hue test for examination of color discrimination. Baltimore, MD: Munsell Color Co.

LAKowskI, R. (1969). Theory and practice of colour vision testing: A review. Part 2. British Journal of Industrial Medicine, 26, 265-288.
LAKOWSKI, R. (1971). Calibration, validation and population norms for the Pickford-Nicolson anomaloscope. British Journal of Physiological Optics, 26, 166-182.

Pokorny, J., Collins, W., Howett, G., Lakowski, R., Lewis, M., Moreland, J., Paulson, H., \& Smith, V. C. (1981). Procedures for testing color vision: Report of working group 41. Washington, DC: National Academy Press.

(Manuscript accepted for publication March 18, 1986.) 